$\begin{array}{cccc}\begin{array}{c}\text { Laboratory } \\ \text { number }\end{array} & \begin{array}{c}\text { Published } \\ \text { reference }\end{array} & \begin{array}{c}\text { Original date or } \\ \text { other value }\end{array} & \begin{array}{c}\text { Corrected date } \\ \text { or other value }\end{array} \\ -21 & 118: 303 & 8120 \pm 240 & \\ -22 & 118: 303 & 5630 \pm 160 & \\ -23 & 118: 303 & 7400 \pm 190 & \\ -24 & 118: 303 & 8150 \pm 210 & \\ -25 & 118: 303 & 3250 \pm 140 & \\ -26 & 128: 110 & 3830 \pm 130 & \\ -27 & 128: 110 & 1350 \pm 140 & \\ -28 & 128: 110 & 7110 \pm 170 & \\ -29 & 128: 110 & 4400 \pm 130 & \\ -30 & 128: 110 & 8490 \pm 200 & \\ -31 & 128: 110 & 6170 \pm 160 & \\ -32 & 128: 111 & 7080 \pm 140 & \\ -33 & 128: 111 & 500 \pm 100 & \end{array}$

A.D./

B.C. date

6170 B.C.

3690 B.C.

5450 B.C.

6200 B.C.

1300 B.C.

1880 B.C.

A.D. 600

5160 B.C.

2450 B.C.

6540 B.C.

4220 B.C.

5130 B.C.

A.D. 1450

${ }^{1}$ All dates reprinted as published in original lists, without further scrutiny. Reference standard is 50 -yr-old pine, probably not significantly different from 0.95 x NBS oxalic acid.

\title{
Trondheim, Norwegian Institute of Technology
}

\begin{tabular}{|c|c|c|c|c|c|}
\hline $\begin{array}{l}\text { Laboratory } \\
\text { number }\end{array}$ & $\begin{array}{l}\text { Published } \\
\text { reference }\end{array}$ & $\begin{array}{l}\text { Original date or } \\
\text { other value }\end{array}$ & $\begin{array}{l}\text { Corrected date } \\
\text { or other value }\end{array}$ & $\partial \mathrm{C}^{14}, \%$ & $\begin{array}{l}\text { A.D./ } \\
\text { B.C. date }\end{array}$ \\
\hline T-23 & $43: 176$ & $2510 \pm 140\}$ & Av $2440 \pm 100$ & $-268 \pm 11\}$ & \\
\hline-26 & $43: 176$ & $2370 \pm 140\}$ & Av. $2440=100$ & $-256 \pm 13 \int$ & 490 B.C. \\
\hline-37 & $41: 79$ & $1190 \pm 60$ & & $-143 \pm 5$ & A.D. 770 \\
\hline-44 & $42: 92$ & $1900 \pm 100$ & & $-205 \pm 10$ & A.D. 60 \\
\hline-45 & $42: 92$ & $1580 \pm 380$ & & $-178 \pm 20$ & A.D. 380 \\
\hline-48 & $42: 92$ & $1600 \pm 360$ & & $-180 \pm 14$ & A.D. 360 \\
\hline-50 & $41: 77$ & $10,500 \pm 400$ & & $-729 \pm 19$ & 8540 B.C. \\
\hline-51 & $42: 85$ & $11,680 \pm 170$ & & $-766 \pm 9$ & 9720 B.C. \\
\hline-53 & $41: 77$ & $10,720 \pm 240$ & & $-737 \pm 17$ & 8760 B.C. \\
\hline-55 & $41: 77$ & $2560 \pm 70$ & & $-273 \pm 8$ & 600 B.C. \\
\hline-80 & $41: 78$ & $8770 \pm 100$ & & $-665 \pm 6$ & 6810 B.C. \\
\hline-81 & $41: 78$ & $9410 \pm 220$ & & $-690 \pm 12$ & 7450 B.C. \\
\hline-82 & $41: 80$ & $1430 \pm 80$ & & $-165 \pm 8$ & A.D. 530 \\
\hline-83 & $41: 80$ & $1410 \pm 75$ & & $-159 \pm 7$ & A.D. 550 \\
\hline-86 & $41: 80$ & $1430 \pm 80$ & & $-163 \pm 9$ & A.D. 530 \\
\hline-87 & $41: 78$ & $9090 \pm 230$ & & $-678 \pm 12$ & 7130 B.C. \\
\hline-88 & $41: 78$ & $8710 \pm 280$ & & $-662 \pm 15$ & 6750 B.C. \\
\hline$-89 A$ & $42: 88$ & $2100 \pm 130$ & & $-224 \pm 14$ & 140 B.C. \\
\hline$-89 B$ & $42: 88$ & $2400 \pm 150$ & & $-262 \pm 12$ & 440 B.C. \\
\hline-90 & $41: 78$ & $4980 \pm 160$ & & $-462 \pm 12$ & 3020 B.C. \\
\hline$-91 \mathrm{~A}$ & $42: 88$ & $3700 \pm 150$ & & $-365 \pm 14$ & 1740 B.C. \\
\hline$-91 B$ & $42: 88$ & $3100 \pm 120$ & & $-316 \pm 11$ & 1140 B.C. \\
\hline-92 & $41: 79$ & $>31,000$ & & $-992 \pm 17$ & \\
\hline .98 & $41: 79$ & $7200 \pm 270$ & & $-592 \pm 15$ & 5240 B.C. \\
\hline-99 & $42: 94$ & $800 \pm 90$ & & $-95 \pm 11$ & A.D. 1160 \\
\hline-100 & $42: 95$ & $800 \pm 100$ & & $-98 \pm 12$ & A.D. 1160 \\
\hline-101 & $42: 95$ & $610 \pm 90$ & & $-75 \pm 11$ & A.D. 1350 \\
\hline-105 & $42: 90$ & $11,700 \pm 300$ & See T-105 bis & & \\
\hline$-105 \mathrm{bis}$ & $43: 161$ & $12,000 \pm 300$ & & $-776 \pm 12$ & 10,050 B.C. \\
\hline-106 & $42: 90$ & $11,600 \pm 300$ & & $-766 \pm 13$ & 9640 B.C. \\
\hline-107 & $42: 91$ & $7780 \pm 190$ & & $-620 \pm 12$ & 5820 B.C. \\
\hline-110 & $42: 85$ & $11,500 \pm 400$ & & $-761 \pm 18$ & 9540 B.C. \\
\hline-111 & $42: 86$ & $10,700 \pm 300$ & See $\mathrm{T}-111$ bis c & & 10,050 B.C. \\
\hline$-111 \mathrm{bis}$ & $43: 161$ & $11,000 \pm 300$ & & $-746 \pm 13$ & 9050 B.C. \\
\hline-112 & $42: 85$ & $11,500 \pm 230$ & & $-761 \pm 11$ & 9540 B.C. \\
\hline .113 & $42: 85$ & $10,200 \pm 350$ & & $-720 \pm 17$ & 8240 B.C. \\
\hline-114 & $144: 284$ & $0 \pm 60$ & & $+8 \pm 8$ & \\
\hline .115 & $144: 284$ & $0 \pm 135$ & & $+1 \pm 14$ & \\
\hline
\end{tabular}




\begin{tabular}{|c|c|c|c|c|c|}
\hline $\begin{array}{c}\text { Laboratory } \\
\text { number }\end{array}$ & $\begin{array}{l}\text { Published } \\
\text { reference }\end{array}$ & $\begin{array}{l}\text { Original date or } \\
\text { other value }\end{array}$ & $\begin{array}{l}\text { Corrected date } \\
\text { or other value }\end{array}$ & $\partial \mathrm{C}^{14}, \%$ & $\begin{array}{l}\text { A.D./ } \\
\text { B.C. date }\end{array}$ \\
\hline-116 & $42: 90$ & $>36,000$ & & $-1004 \pm 13$ & \\
\hline-117 & $42: 87$ & $9950 \pm 300$ & & $-710 \pm 13$ & 7910 B.C. \\
\hline-118 & $42: 86$ & $9850 \pm 350$ & See T-118 bis $d$ & & \\
\hline$-118 \mathrm{bis}$ & $43: 161$ & $10,050 \pm 350$ & & $-713 \pm 14$ & 8100 B.C. \\
\hline$-119 \mathrm{~A}$ & $42: 87$ & $9250 \pm 250$ & & $-683 \pm 12$ & 7290 B.C. \\
\hline$-119 B$ & $42: 87$ & $7300 \pm 200$ & & $-590 \pm 12$ & 5340 B.C. \\
\hline$-119 \mathrm{C}$ & $42: 87$ & $9450 \pm 250$ & $9700 \pm 250 \mathrm{c}$ & $-700 \pm 12$ & 7490 B.C. \\
\hline-120 & $42: 87$ & $9100 \pm 180$ & & $-678 \pm 12$ & 7140 B.C. \\
\hline-121 & $42: 87$ & $6570 \pm 150$ & & $-558 \pm 12$ & 4610 B.C. \\
\hline-122 & $42: 87$ & $660 \pm 100$ & See T-122 bis c & & \\
\hline$-122 \mathrm{bis}$ & $43: 161$ & $980 \pm 100$ & & $-116 \pm 12$ & A.D. 970 \\
\hline-123 & $42: 87$ & $6850 \pm 170$ & & $-573 \pm 12$ & 4890 B.C. \\
\hline-124 & $42: 86$ & $10,300 \pm 250$ & & $-722 \pm 10$ & 8340 B.C. \\
\hline-125 & $42: 86$ & $9880 \pm 240$ & & $-708 \pm 13$ & 7920 B.C. \\
\hline-126 & $42: 86$ & $4820 \pm 160$ & & $-452 \pm 13$ & 2860 B.C. \\
\hline-127 & $42: 92$ & $3410 \pm 140$ & & $-346 \pm 10$ & 1450 B.C. \\
\hline-128 & $42: 92$ & $3970 \pm 100$ & & $-390 \pm 9$ & 2010 B.C. \\
\hline-129 & $42: 92$ & $4130 \pm 120$ & & $-402 \pm 10$ & 2170 B.C. \\
\hline-130 & $42: 93$ & $6100 \pm 140$ & & $-532 \pm 10$ & 4140 B.C. \\
\hline-131 & $42: 93$ & $6860 \pm 140$ & & $-574 \pm 9$ & 4900 B.C. \\
\hline-132 & $42: 93$ & $1120 \pm 90$ & & $-130 \pm 10$ & A.D. 840 \\
\hline-133 & $42: 93$ & $4700 \pm 120$ & & $-443 \pm 10$ & 2740 B.C. \\
\hline-134 & $42: 93$ & $2220 \pm 110$ & & $-241 \pm 12$ & 260 B.C. \\
\hline-136 & $42: 94$ & $2220 \pm 110$ & & $-241 \pm 10$ & 260 B.C. \\
\hline-137 & $42: 95$ & $2010 \pm 110$ & & $-222 \pm 11$ & 50 B.C. \\
\hline-138 & $42: 88$ & $12,200 \pm 350$ & & $-781 \pm 11$ & 10,240 B.C. \\
\hline-139 & $42: 88$ & $12,700 \pm 350$ & & $-794 \pm 11$ & 10,740 B.C. \\
\hline-140 & $42:$ & $34,000 \pm 3000$ & & $-986 \pm 10$ & 32,040 B.C. \\
\hline-141 & $42: 89$ & $5250 \pm 150$ & & $-480 \pm 11$ & 3290 B.C. \\
\hline-142 & $42: 89$ & $11,500 \pm 300$ & & $--760 \pm 13$ & 9540 B.C. \\
\hline-143 & $42: 89$ & $>27,000$ & & $-999 \pm 28$ & \\
\hline-146 & $42: 91$ & $>44,000$ & & $-1000 \pm 5$ & \\
\hline-148 & $42: 89$ & $>23,000$ & & $-976 \pm 25$ & \\
\hline$-149 \mathrm{~A}$ & $42: 90$ & $13,000 \pm 400$ & & $-802 \pm 11$ & 11,040 B.C. \\
\hline$-149 \mathrm{~B}$ & $42: 90$ & $13,150 \pm 300$ & & $-806 \pm 9$ & 11,190 B.C. \\
\hline-150 & $42: 90$ & $12,650 \pm 300$ & & $-793 \pm 9$ & 10,690 B.C. \\
\hline-151 & $42: 90$ & $11,300 \pm 300$ & & $-756 \pm 9$ & 9340 B.C. \\
\hline-152 & $42: 90$ & $10,800 \pm 300$ & & $-739 \pm 11$ & 8840 B.C. \\
\hline-153 & $42: 91$ & $9000 \pm 200$ & & $-674 \pm 10$ & 7040 B.C. \\
\hline-156 & $42: 91$ & $6420 \pm 160$ & & $-551 \pm 12$ & 4460 B.C. \\
\hline-157 & $43: 177$ & $530 \pm 100$ & & $-68 \pm 10$ & A.D. 1420 \\
\hline-160 & $42: 91$ & $>39,000$ & & $-998 \pm 6$ & \\
\hline-162 & $42: 94$ & $3670 \pm 130$ & & $-367 \pm 11$ & 1710 B.C. \\
\hline-163 & $42: 94$ & $3860 \pm 140$ & & $-382 \pm 11$ & 1900 B.C. \\
\hline-161 & $42: 95$ & $950 \pm 80$ & & $-136 \pm 8$ & A.D. 1010 \\
\hline-165 & $42: 95$ & $1980 \pm 100$ & & $-219 \pm 10$ & 20 B.C. \\
\hline-168 & $42: 91$ & $12,550 \pm 200$ & & $-790 \pm 8$ & 10,590 B.C. \\
\hline-169 & $43: 179$ & $>32,500$ & & $-989 \pm 10$ & \\
\hline-170 & $43: 179$ & $>39,000$ & & $-996 \pm 9$ & \\
\hline-171 & $43: 179$ & $>33,000$ & & $-988 \pm 9$ & \\
\hline-172 & $43: 179$ & $>38,000$ & & $-996 \pm 9$ & \\
\hline$-178 \mathrm{bis}$ & $43: 161$ & $10,200 \pm 220$ & & $-720 \pm 11$ & 8250 B.C. \\
\hline$-178 \mathrm{bis}$ & $43: 168$ & $10,200 \pm 220$ & & $-720 \pm 9$ & 8250 B.C. \\
\hline-179 & $43: 168$ & $9750 \pm 250$ & & $-706 \pm 9$ & 7800 B.C. \\
\hline$-180 \mathrm{bis}$ & $43: 162$ & $9200 \pm 300$ & & $-682 \pm 14$ & 7250 B.C. \\
\hline$-180 \mathrm{bis}$ & $43: 168$ & $9250 \pm 300$ & & $-682 \pm 14$ & 7300 B.C. \\
\hline -181bis & $43: 168$ & $2050 \pm 150$ & & $--226 \pm 14$ & 100 B.C. \\
\hline -181bis & $43: 162$ & $2050 \pm 150$ & & $-226 \pm 15$ & 100 B.C. \\
\hline-182 & $43: 165$ & $7750 \pm 150$ & & $-621 \pm 10$ & 5800 B.C. \\
\hline-183 & $43: 162$ & $5500 \pm 150$ & & $-495 \pm 12$ & 3550 B.C. \\
\hline-184 & $43: 162$ & $5700 \pm 150$ & & $-513 \pm 11$ & 3750 B.C. \\
\hline
\end{tabular}




\begin{tabular}{|c|c|c|c|c|c|}
\hline $\begin{array}{l}\text { Laboratory } \\
\text { number }\end{array}$ & $\begin{array}{l}\text { Published } \\
\text { reference }\end{array}$ & $\begin{array}{l}\text { Original date or } \\
\text { other value }\end{array}$ & $\begin{array}{l}\text { Corrected date } \\
\text { or other value }\end{array}$ & $\partial \mathrm{C}^{14}, \% 0$ & $\begin{array}{c}\text { A.D./ } \\
\text { B.C. date }\end{array}$ \\
\hline-185 & $43: 163$ & $6350 \pm 150$ & & $-545 \pm 11$ & 4400 B.C. \\
\hline-186 & $43: 163$ & $4100 \pm 100$ & & $-399 \pm 8$ & 2150 B.C. \\
\hline-187 & $43: 166$ & $10,350 \pm 300$ & & $-725 \pm 10$ & 8400 B.C. \\
\hline -187bis & $43: 162$ & $10,350 \pm 300$ & & $-725 \pm 10$ & 8400 B.C. \\
\hline-188 & $144: 288$ & $1750 \pm 100$ & & $-194 \pm 12$ & A.D. 200 \\
\hline-189 & $144: 288$ & $2100 \pm 100$ & & $-228 \pm 9$ & 150 B.C. \\
\hline-190 & $144: 287$ & $2450 \pm 100$ & & $-261 \pm 11$ & 500 B.C. \\
\hline-192 & $43: 176$ & $2100 \pm 150$ & & $-230 \pm 11$ & 150 B.C. \\
\hline-193 & $144: 289$ & $530 \pm 70$ & & $-65 \pm 8$ & A.D. 1420 \\
\hline-194 & $144: 290$ & $470 \pm 70$ & & $-51 \pm 8$ & A.D. 1480 \\
\hline-195 & $43: 178$ & $1330 \pm 70$ & $1260 \pm 70 \mathrm{c}$ & & A.D. 690 \\
\hline$-195 \mathrm{~A}$ & $43: 178$ & $1310 \pm 80$ & $1230 \pm 80 \mathrm{c}$ & $-140 \pm 10$ & A.D. 720 \\
\hline$-195 \mathrm{~B}$ & $43: 178$ & $1380 \pm 120$ & & $-155 \pm 12$ & A.D. 570 \\
\hline-196 & $43: 173$ & $4750 \pm 150$ & & $-449 \pm 9$ & 2800 B.C. \\
\hline-198 & $43: 174$ & $3650 \pm 150$ & & $-367 \pm 11$ & 1700 B.C. \\
\hline-204 & $43: 177$ & $1050 \pm 100$ & & $-125 \pm 12$ & A.D. 900 \\
\hline-205 & $43: 177$ & $800 \pm 100$ & & $-98 \pm 12$ & A.D. 1150 \\
\hline .206 & $43: 177$ & $900 \pm 100$ & & $-109 \pm 11$ & A.D. 1050 \\
\hline-207 & $43: 177$ & $900 \pm 100$ & & $-110 \pm 11$ & A.D. 1050 \\
\hline-208 & $43: 171$ & $7950 \pm 170$ & & $-629 \pm 9$ & 6000 B.C. \\
\hline-209 & $43: 170$ & $7400 \pm 250$ & & $-602 \pm 13$ & 5450 B.C. \\
\hline .214 & $43: 167$ & $11,400 \pm 250$ & $11,300 \pm 250 \mathrm{c}$ & $-755 \pm 11$ & 9350 B.C. \\
\hline-215 & $144: 289$ & $8150 \pm 200$ & & $-640 \pm 10$ & 6200 B.C. \\
\hline-216 & $144: 288$ & $5900 \pm 150$ & & $-523 \pm 10$ & 3950 B.C. \\
\hline-217 & $144: 289$ & $2100 \pm 100$ & & $-236 \pm 9$ & 150 B.C. \\
\hline-223 & $43: 168$ & $11,200 \pm 200$ & & $-753 \pm 7$ & 9250 B.C. \\
\hline-227 & $43: 175$ & $2400 \pm 200$ & & $-256 \pm 17$ & 450 B.C. \\
\hline$-228 \mathrm{~A}$ & $144: 283$ & $11,700 \pm 150$ & & $-767 \pm 6$ & 9750 B.C. \\
\hline-229 & $43: 171$ & $10,150 \pm 300$ & & $-718 \pm 18$ & 8200 B.C. \\
\hline-231 & $43: 169$ & $8700 \pm 200$ & $8900 \pm 200 \mathrm{c}$ & $-669 \pm 9$ & 6950 B.C. \\
\hline-234 & $43: 174$ & $3850 \pm 100$ & & $-380 \pm 10$ & 1900 B.C. \\
\hline-235 & $43: 174$ & $4550 \pm 150\}$ & Av. $4600 \pm 110$ & $-435 \pm 10\}$ & 2650 B.C. \\
\hline-236 & $43: 174$ & $4650 \pm 150\}$ & & $-448 \pm 11\}$ & 2650 B.C. \\
\hline-237 & $43: 174$ & $2600 \pm 150$ & & $-277 \pm 12$ & 650 B.C. \\
\hline-240 & $43: 177$ & $1350 \pm 80$ & & & A.D. 600 \\
\hline$-240 \mathrm{~A}$ & $43: 178$ & $1400 \pm 100$ & & $-159 \pm 10$ & A.D. 550 \\
\hline$-240 \mathrm{~B}$ & $43: 178$ & $1300 \pm 100$ & & $-149 \pm 9$ & A.D. 650 \\
\hline $.241 \mathrm{~A}$ & $43: 170$ & $7900 \pm 250$ & & $-626 \pm 11$ & 5950 B.C. \\
\hline$-241 \mathrm{~B}$ & $43: 170$ & $8000 \pm 300$ & & $-633 \pm 18$ & 6050 B.C. \\
\hline-243 & $43: 163$ & $2450 \pm 100$ & & $-259 \pm 12$ & 500 B.C. \\
\hline$-24,4$ & $43: 163$ & $4100 \pm 150$ & & $-401 \pm 11$ & 2150 B.C. \\
\hline-245 & $43: 164$ & $850 \pm 80$ & & $-102 \pm 9$ & A.D. 1100 \\
\hline-246 & $43: 166$ & $10,550 \pm 250$ & & $-731 \pm 11$ & 8600 B.C. \\
\hline$-249 \mathrm{~B}$ & $43: 172$ & $11,500 \pm 300$ & & $-761 \pm 11$ & 9550 B.C. \\
\hline-250 & $43: 175$ & $1350 \pm 80$ & & $-166 \pm 9$ & A.D. 600 \\
\hline-252 & $43: 175$ & $1500 \pm 100$ & & $-177 \pm 10$ & A.D. 450 \\
\hline-253 & $43: 175$ & $1400 \pm 100$ & & $-165 \pm 9$ & A.D. 550 \\
\hline .254 & $43: 175$ & $1600 \pm 100$ & & $-184 \pm 10$ & A.D. 350 \\
\hline-255 & $43: 175$ & $1350 \pm 100$ & & $-156 \pm 10$ & A.D. 600 \\
\hline-256 & $144: 289$ & $6850 \pm 150$ & & $-573 \pm 9$ & 4900 B.C. \\
\hline-257 & $144: 289$ & $6550 \pm 200$ & $7550 \pm 200 \mathrm{c}$ & $-609 \pm 8$ & 5600 B.C. \\
\hline-258 & $43: 175$ & $3550 \pm 150$ & & $-355 \pm 10$ & 1600 B.C. \\
\hline-259 & $43: 175$ & $980 \pm 110$ & & $-114 \pm 13$ & A.D. 970 \\
\hline-260 & $144: 289$ & $6250 \pm 150$ & & $-540 \pm 7$ & 4300 B.C. \\
\hline-261 & $43: 170$ & $11,000 \pm 250$ & & $-746 \pm 10$ & 9050 B.C. \\
\hline-266 & $43: 164$ & $4500 \pm 150$ & & $-428 \pm 15$ & 2550 B.C. \\
\hline-267 & $43: 164$ & $6250 \pm 200$ & & $-540 \pm 12$ & 4300 B.C. \\
\hline-269 & $43: 167$ & $12,300 \pm 250$ & & $-784 \pm 10$ & 10,350 B.C. \\
\hline-270 & $43: 165$ & $7400 \pm 150$ & & $-602 \pm 9$ & 5450 B.C. \\
\hline-271 & $43: 165$ & $10,600 \pm 250$ & & $-733 \pm 17$ & 8650 B.C. \\
\hline$-271 B$ & $144: 281$ & $10,950 \pm 130$ & & $-745 \pm 7$ & 9000 B.C. \\
\hline
\end{tabular}


Radiocarbon Measurements:

\begin{tabular}{|c|c|c|c|c|c|}
\hline $\begin{array}{c}\text { Laboratory } \\
\text { number }\end{array}$ & $\begin{array}{l}\text { Published } \\
\text { reference }\end{array}$ & $\begin{array}{l}\text { Original date or } \\
\quad \text { other value }\end{array}$ & $\begin{array}{l}\text { Corrected date } \\
\text { or other value }\end{array}$ & $\partial \mathrm{C}^{14}, \% 0$ & $\begin{array}{l}\text { A.D./ } \\
\text { B.C. date }\end{array}$ \\
\hline-272 & $144: 283$ & $8600 \pm 150$ & & $-658 \pm 10$ & 6650 B.C. \\
\hline-273 & $43: 171$ & $8300 \pm 250$ & & $-644 \pm 14$ & 6350 B.C. \\
\hline-274 & $43: 176$ & $900 \pm 140$ & & $-114 \pm 12$ & A.D. 1050 \\
\hline-275 & $43: 177$ & $920 \pm 100$ & & $-109 \pm 12$ & A.D. 1030 \\
\hline-276 & $43: 176$ & $1550 \pm 100$ & & $-170 \pm 10$ & A.D. 400 \\
\hline-279 & $144: 286$ & $6900 \pm 150$ & & $-577 \pm 10$ & 4950 B.C. \\
\hline-280 & $144: 286$ & $9150 \pm 200$ & & $-689 \pm 10$ & 7200 B.C. \\
\hline-281 & $43: 171$ & $3050 \pm 100$ & & $-317 \pm 10$ & 1100 B.C. \\
\hline-285 & $43: 173$ & $2600 \pm 100$ & & $-277 \pm 15$ & 650 B.C. \\
\hline-286 & $43: 169$ & $9500 \pm 200$ & & $-695 \pm 9$ & 7550 B.C. \\
\hline-287 & $43: 169$ & $9050 \pm 200$ & & $-677 \pm 18$ & 7100 B.C. \\
\hline .290 & $43: 169$ & $8200 \pm 200$ & & $-644 \pm 18$ & 6250 B.C. \\
\hline-291 & $43: 169$ & $7250 \pm 200$ & & $-595 \pm 18$ & 5300 B.C. \\
\hline-292 & $43: 170$ & $6800 \pm 170$ & & $--572 \pm 14$ & 4850 B.C. \\
\hline-293 & $43: 172$ & $8250 \pm 250$ & & $-642 \pm 16$ & 6300 B.C. \\
\hline-294 & $43: 165$ & $9900 \pm 200$ & $9800 \pm 200 \mathrm{c}$ & $-708 \pm 11$ & 7850 B.C. \\
\hline-295 & $43: 165$ & $9450 \pm 250$ & & $-693 \pm 18$ & 7500 B.C. \\
\hline-296 & $43: 166$ & $6100 \pm 150$ & & $-537 \pm 15$ & 4150 B.C. \\
\hline-297 & $43: 179$ & $11,450 \pm 250$ & & $-758 \pm 14$ & 9500 B.C. \\
\hline-298 & $43: 180$ & $33,500 \pm 1000$ & & $-983 \pm 9$ & 31,550 B.C \\
\hline-299 & $43: 180$ & $>39,000$ & & $-996 \pm 8$ & \\
\hline-302 & $144: 284$ & $10,250 \pm 440$ & & $-723 \pm 20$ & 8300 B.C. \\
\hline-304 & $43: 171$ & $10,050 \pm 250$ & & $-715 \pm 18$ & 8100 B.C. \\
\hline-305 & $43: 172$ & $11,700 \pm 230$ & & $-759 \pm 18$ & 9750 B.C. \\
\hline-315 & $43: 170$ & $10,700 \pm 300$ & & $-736 \pm 20$ & 8750 B.C. \\
\hline-316 & $43: 167$ & $11,700 \pm 250$ & & $-767 \pm 13$ & 9750 B.C. \\
\hline-329 & $144: 288$ & $2600 \pm 60$ & & $-278 \pm 6$ & 650 B.C. \\
\hline-330 & $144: 288$ & $2660 \pm 80$ & & $-282 \pm 8$ & 710 B.C. \\
\hline-331 & $144: 288$ & $2620 \pm 80$ & & $-278 \pm 8$ & 670 B.C. \\
\hline$-338 \mathrm{~A}$ & $144: 286$ & $8580 \pm 110$ & & $-656 \pm 7$ & 6630 B.C. \\
\hline$-338 \mathrm{~B}$ & $144: 286$ & $9240 \pm 130$ & & $-683 \pm 7$ & 7290 B.C. \\
\hline$-340 \mathrm{~B}$ & $144: 287$ & $21,350 \pm 350$ & & $-980 \pm 7$ & 19,400 B.C. \\
\hline$-34: 1$ & $144: 287$ & $7000 \pm 100$ & & $-582 \pm 7$ & 5050 B.C. \\
\hline-343 & $144: 285$ & $7970 \pm 180$ & & $-630 \pm 9$ & 6020 B.C. \\
\hline-350 & $144: 281$ & $2200 \pm 100$ & & $-240 \pm 10$ & 250 B.C. \\
\hline-351 & $144: 280$ & $3070 \pm 100$ & & $-318 \pm 9$ & 1120 B.C. \\
\hline-353 & $144: 282$ & $3470 \pm 100$ & & $-351 \pm 8$ & 1520 B.C. \\
\hline-361 & $144: 287$ & $7310 \pm 120$ & & $-598 \pm 7$ & 5360 B.C. \\
\hline-362 & $144: 285$ & $9930 \pm 190$ & & $-705 \pm 10$ & 7980 B.C. \\
\hline-363 & $144: 285$ & $3300 \pm 110$ & & $--337 \pm 10$ & 1350 B.C. \\
\hline-370 & $144: 284$ & $4090 \pm 150$ & & $-402 \pm 12$ & 2140 B.C. \\
\hline$-378 \mathrm{~B}$ & $144: 283$ & $11,480 \pm 260$ & & $-761 \pm 10$ & 9530 B.C. \\
\hline-379 & $144: 283$ & $11,430 \pm 260$ & & $-758 \pm 11$ & 9480 B.C. \\
\hline-380 & $144: 282$ & $9660 \pm 210$ & & $-700 \pm 10$ & 7710 B.C. \\
\hline-381 & $144: 281$ & $9890 \pm 240$ & & $-710 \pm 10$ & 7940 B.C. \\
\hline-382 & $144: 282$ & $10,940 \pm 240$ & & $-740 \pm 9$ & 8990 B.C. \\
\hline-385 & $144: 281$ & $2710 \pm 90$ & & $-287 \pm 8$ & 760 B.C. \\
\hline-390 & $144: 283$ & $10,310 \pm 180$ & & $-720 \pm 9$ & 8360 B.C. \\
\hline-391 & $144: 288$ & $4590 \pm 110$ & & $-433 \pm 10$ & 2640 B.C. \\
\hline-392 & $144: 288$ & $4400 \pm 100$ & & $-422 \pm 8$ & 2450 B.C. \\
\hline-394 & $144: 286$ & $770 \pm 90$ & & $--92 \pm 9$ & A.D. 1180 \\
\hline
\end{tabular}

